\title{
Pkw-Nutzung im Wandel des Geschlechterverhältnisses - Trends über drei Jahrzehnte
}

\author{
Kathrin Konrad $^{1}$ Joachim Scheiner ${ }^{2}$ Christian Holz-Rau ${ }^{2}$
}

Eingegangen: 17. Juli 2015 / Angenommen: 26. Mai 2016 / Online publiziert: 28. Juni 2016

(c) Springer-Verlag Berlin Heidelberg 2016

Zusammenfassung Dieser Beitrag beantwortet die Frage, wie sich in Deutschland in den vergangenen Jahrzehnten vor dem Hintergrund eines sich wandelnden Geschlechterverhältnisses die Pkw-Nutzung von Männern und Frauen entwickelt hat. Außerdem wird beantwortet, wie sich statistische Unterschiede hinsichtlich der Pkw-Nutzung von Frauen und Männern erklären lassen. Anhand der deutschen Mobilitätserhebungen KONTIV und MiD wird für den Zeitraum 1976 bis 2008 ein langfristiger Trend der geschlechtsspezifischen Pkw-Nutzung nachgezeichnet und in den weiteren Kontext gesellschaftlicher Veränderungen eingeordnet. Die Analysen ermöglichen auch eine Annäherung an die Frage, inwiefern geschlechtsspezifische Unterschiede bezüglich der Pkw-Nutzung Resultat sozialer Rollen (z. B. Erwerbstätigkeit), Restriktionen (geringere Pkw-Verfügbarkeit der Frauen), rational-ökonomischer Haushaltsentscheidungen über eine knappe Ressource Pkw oder unterschiedlicher Normen und Präferenzen sind. Differenzierte Analysen zeigen, wie sich die Pkw-Nutzung von Männern und Frauen mit verschiedenen restriktiven Rahmenbe-

Dr.-Ing. Kathrin Konrad

kathrin.konrad@ils-forschung.de

Prof. Dr. Joachim Scheiner

joachim.scheiner@tu-dortmund.de

Prof. Dr.-Ing. Christian Holz-Rau

christian.holz-rau@tu-dortmund.de

1 Forschungsgruppe Alltagsmobilität und Verkehrssysteme, ILS Institut für Landes- und Stadtentwicklungsforschung gGmbH, Brüderweg 22-24, 44135 Dortmund, Deutschland

2 Fachgebiet Verkehrswesen und Verkehrsplanung, Technische Universität Dortmund, August-Schmidt-Straße 10, 44221 Dortmund, Deutschland dingungen der Mobilität (Teil-/Vollmotorisierung des Haushalts, Gemeindegrößenklassen) und verschiedenen soziodemographischen Merkmalen (z. B. Haushaltskontext, Erwerbstätigkeit) entwickelt hat. Im Wesentlichen konvergiert die Pkw-Nutzung zwischen Männern und Frauen durch ein „Aufholen“ der Frauen. In Haushalten, in denen der Pkw eine knappe Ressource darstellt, ist aber auch eine Umverteilung des Pkw zwischen Männern und Frauen zu beobachten. Zwischen verschiedenen Bevölkerungsgruppen unterscheidet sich dieser Trend allerdings: Beispielsweise nimmt die Pkw-Nutzung der Frauen in Klein- und Mittelstädten deutlich stärker zu als in Großstädten. Die Ergebnisse deuten außerdem auf geschlechtsspezifische Normen oder Präferenzen hin, denn selbst bei gleichen Rahmenbedingungen wie Vollzeiterwerbstätigkeit und uneingeschränkter PkwVerfügbarkeit fahren die Frauen seltener Auto als Männer.

Schlüsselwörter Pkw-Nutzung · Geschlechterverhältnis · Mobilitätstrends · Präferenzen · Motorisierung

\section{Car driving in the context of changing gender relations - trends over three decades}

Abstract This paper aims at answering the question, how the usage of cars both of men and women has changed in Germany in the context of changing gender relations during the last decades. Moreover, gender-specific differences in car usage are pointed out and their root causes are analysed based on German Travel Survey data. This is achieved by tracing the development of gender-specific car usage between 1976 and 2008 and interpreting the trends identified in a wider context of societal changes. The analyses conducted give results on whether gender-specific car usage results from social roles (e. g. labour participation), restric- 
tions (e.g. lower car availability of women), economically rational household decisions on the car as a restricted resource, or gender-specific preferences. Beyond that it is shown how car usage has changed with respect to men and women with different characteristics (e.g. household motorization, labour participation, household type, municipality size). The results given in the paper show that convergence of men's and women's car usage predominantly results from an increasing women's car usage. Regarding households where the number of drivers exceeds the number of available cars, i. e. the available cars are shared, we find a re-allocation of the scarce resource car between men and women. Yet, this trend varies: For example, women's car usage increases stronger in small and in medium municipalities than in big cities. Furthermore, the results hint at gender-specific preferences: Even under very similar conditions such as full-time employment and unrestricted car availability, women use the car less often than men.

Keywords Car use - Gender relation - Mobility trends · Preferences · Motorization

\section{Einleitung}

In den vergangenen Jahrzehnten vollzogen sich in Deutschland vielschichtige Veränderungen: Beispiele sind der demographische Wandel, Sub- und Reurbanisierung und Umstrukturierungen des Arbeitsmarktes. Ebenso wandeln sich Normen und Werte, es ist beispielsweise ,zu einer Höherbewertung von Selbstentfaltungswerten [...] gekommen“ (Schubarth 2010: 25). Hiermit gehen eine Individualisierung und Pluralisierung der Gesellschaft, von Lebensstilen und Haushaltsformen einher (vgl. z. B. Beck 1986; Hurrelmann 2012: 60).

Eine zentrale Dimension dieses Wandels ist der Wandel des Geschlechterverhältnisses. Laut Meuser (2008: 640) verliert ,die Geschlechtszugehörigkeit an Bedeutung für die soziale Positionierung“. So wandeln sich Geschlechternormen, Geschlechterrollen, die geschlechtsspezifische Arbeitsteilung (vgl. Meuser 2009: 148; BMFSFJ 2011: 109; Klammer 2012: 165; Maiwald 2012: 912; Peuckert 2012: 414 f.) und die Alltagsmobilität von Männern und Frauen (Scheiner 2009: 75 ff.). Gleichzeitig wird die ,,weiterhin bestehende Geltung traditionaler Geschlechternormen“ (Liebig/Sauer/Schupp 2011: 40) sowie traditionaler Geschlechterrollen und Arbeitsteilung (z. B. Anger/Kottwitz 2009: 102 ff.; BMFSFJ 2011: 174) betont.

Zur Erklärung geschlechtsspezifischer Unterschiede in der Arbeitsteilung und Alltagsmobilität finden rollen-, präferenz- und restriktionstheoretische sowie rational-ökonomische Ansätze und das Konzept patriarchalischer Macht- verhältnisse Anwendung. Sie werden hinsichtlich ihrer Gültigkeit jedoch eher selten explizit empirisch geprüft.

Dieser Beitrag stellt folgende Fragen: Wie verändert sich vor dem Hintergrund des Wandels des Geschlechterverhältnisses die Alltagsmobilität von Frauen und Männern und welche Erklärungskraft haben die verschiedenen theoretischen Ansätze ihres Verkehrsverhaltens? Der Fokus zur Beantwortung dieser Fragestellungen liegt auf dem Autofahren. Die Verfügbarkeit und Nutzung eines Autos ist stark durch Restriktionen, soziale Rollen und Haushaltsentscheidungen bestimmt, laut einigen Studien auch durch mobilitätsbezogene Präferenzen (Götz 2011). Die durchgeführten Analysen erlauben neben der Abbildung langfristiger Trends auch Aussagen zur Relevanz der zuvor genannten Erklärungsansätze für geschlechtsspezifische Unterschiede im Verkehrsverhalten.

Zunächst wird in Abschnitt 2 der Stand der Forschung mit einem Fokus auf die Verkehrsmittel- bzw. Pkw-Nutzung einschließlich der theoretischen Erklärungsansätze für diese im Folgenden ,geschlechtsspezifisch“ genannten Unterschiede dargestellt. Dabei werden Forschungslücken definiert, vor deren Hintergrund die Forschungsfragen dieses Beitrags formuliert werden. Anschließend (Abschn. 3) werden die verwendete Datenbasis und Analysemethodik erläutert, die zur Beantwortung der Forschungsfragen zum Einsatz kommen. Die Empirie dieses Beitrags beruht auf Regressionsanalysen, die anhand der KONTIV ${ }^{1}$ und MiD $^{2}$-Daten der Jahre 1976 bis 2008 durchgeführt wurden. Diese Mobilitätsdaten machen einen Zeitraum von 32 Jahren nachvollziehbar. Differenzierte Analysen (Abschn. 4) zeigen geschlechtsspezifische Unterschiede der Pkw-Nutzung, langfristige Trends in unterschiedlichen Bevölkerungsgruppen und ermöglichen Einschätzungen zur Relevanz theoretischer Erklärungsansätze hinsichtlich geschlechtsspezifischer Unterschiede bei der Pkw-Nutzung. Abschließend (Abschn. 5) werden die Ergebnisse zusammengefasst, im Hinblick auf die Forschungsfragen eingeordnet und ein Ausblick gegeben.

\section{Stand der Forschung und Forschungsfragen}

Geschlechterdifferenzen in der Alltagsmobilität wurden seit dem Ende der 1970er-Jahre zu einem wichtigen Forschungsfeld (vgl. Rosenbloom 1978). Generell zeigen entsprechende Studien, dass Frauen mehr Wege zu Zwecken der Haushalts- und Familienarbeit (Einkauf, Besorgungen, Kinderbegleitung), aber weniger Erwerbswege unternehmen als Männer, kürzere Distanzen zurücklegen, ihre Wege

\footnotetext{
1 Kontinuierliche Erhebung zum Verkehrsverhalten.

2 Mobilität in Deutschland; vgl. www.mobilitaet-in-deutschland.de (21.6.2016)
} 
zu komplexeren Wegeketten kombinieren und langsamere Verkehrsmittel nutzen (zu Fuß und öffentliche Verkehrsmittel statt Pkw) (Rutherford/Wekerle 1988; Meyer 1999; Polk 2004; Best/Lanzendorf 2005; Hjorthol 2008; Nobis 2008; Scheiner/Holz-Rau 2012). Eine zentrale Rolle in dieser Forschung spielt die Nutzung des Pkw als Fahrerin/Fahrer, da hiermit einerseits große Freiheit und Flexibilität der Mobilität sowie umfassende Erreichbarkeit, andererseits aber auch männliche Technikaffinität und Technikbeherrschung sowie Macht verbunden werden (Polk 1998). Daneben stellt der Pkw in hochmotorisierten Gesellschaften das dominante Verkehrsmittel dar und gilt als zentrales Problem in der Diskussion um eine nachhaltige Verkehrsgestaltung.

Auch aus Deutschland liegen zur Verkehrsmittelnutzung von Männern und Frauen zahlreiche Studien insbesondere seit den 1990er-Jahren vor. Vor allem die frühen Studien weisen eine deutlich geschlechtsspezifische Verkehrsmittelnutzung aus. Sie zeigen, dass Frauen häufiger als Männer den ÖPNV (Öffentlichen Personennahverkehr) nutzen und zu Fuß gehen, seltener Auto fahren und wenn sie das Auto nutzen, dann eher als Mitfahrerin denn als Selbstfahrerin (Flade 1999: 137; Meyer 1999: 25 f.).

Einige Analysen differenzieren innerhalb der Geschlechtergruppen. Beispielsweise zeigt Valley (2003: 64 ff.) für Deutschland Anfang der 1990er-Jahre, dass Mütter seltener als Frauen ohne Kind Auto fahren, vor allem aufgrund ihrer geringeren Erwerbsbeteiligung und - damit zusammenhängend - niedrigeren Pkw-Verfügbarkeit. Aktuellere Studien finden demgegenüber heraus, dass inzwischen Mütter häufiger als Frauen ohne Kinder Auto fahren (Vance/Iovanna 2007: 10 für Deutschland; Hjorthol 2008: 204 f. für Norwegen). Hingegen nutzen Väter seltener als Männer ohne Kinder das Auto (Vance/Iovanna 2007: 10). Auch weitere Merkmale wie das Alter, das Einkommen und der Wohnort sind relevant für die geschlechtsspezifische Verkehrsmittelnutzung. Die Autonutzung von Männern und Frauen ist in den jüngeren Altersgruppen, höheren Einkommensgruppen und im ländlichen und suburbanen Raum relativ ähnlich (vgl. Valley 2003: 71). Best und Lanzendorf (2005: 115) weisen darauf hin, dass die geschlechtsspezifische Autonutzung wesentlich durch die Unterschiede in der PkwVerfügbarkeit bestimmt wird, die speziell bei älteren Frauen niedrig ist.

Längerfristige Trendanalysen zu geschlechterspezifischen Trends der Mobilität gibt es kaum. Für Norwegen zeichnet Hjorthol (2008: 201) zwischen 1992 und 2000 eine leichte Konvergenz nach, die aus einem stärkeren Bedeutungsgewinn des Autos bei den Frauen resultiert. Trotz solcher Angleichungstendenzen zeigen auch aktuelle Studien noch eine stärker autogeprägte Alltagsmobilität von Männern (vgl. Vance/Iovanna 2007: 10; Nobis 2008: 120 ff. für Deutschland; van Acker/Witlox 2011: 477 für Belgien). In der Shell Pkw-Studie 2014 wird das Aufholen der
Frauen in Sachen Auto so begründet, dass ,in den letzten Jahren die zunehmende Erwerbstätigkeit von Frauen deren Aufholeffekt in Bezug auf Pkw- und Führerschein-Besitz erheblich befördert. Weil Frauen mehr und öfter arbeiten, besitzen sie auch öfter einen Pkw selbst" (Shell Deutschland Oil 2014: 19). Zwischen 1991 und 2004 haben sich die Erwerbstätigenquoten von Männern und Frauen zwar durch einen minimalen Anstieg bei den Frauen und einen schwachen Rückgang bei den Männern leicht angenähert, durch die häufige Teilzeiterwerbstätigkeit und geringfügige Beschäftigung der Frauen bleibt ihre Erwerbsarbeitszeit jedoch konstant. So stieg unter den erwerbstätigen Frauen die Teilzeitquote von 30 auf 42 Prozent, bei den Männern von 2 auf 6 Prozent (vgl. Dressel 2005: 106; Schäfer/Vogel 2005: 132).

Erklärungsansätze für geschlechtsspezifische Unterschiede in der Verkehrsmittelnutzung gibt es aber noch deutlich mehr wie beispielsweise rollen-, präferenz- und restriktionstheoretische aber auch rational-ökonomische Ansätze. Diese Erklärungstheorien sind jedoch nicht als trennscharf zu verstehen (vgl. Scheiner/Holz-Rau 2012: $251 \mathrm{ff}$.).

Präferenztheoretische Ansätze nennen geschlechtsspezifische Präferenzen, Normen und Einstellungen als einen Grund für geschlechtsspezifische Verkehrsmittelnutzungen. Beispielsweise stellen Matthies, Kuhn und Klöckner (2002: 171) eine stärkere ökologische Orientierung bei Frauen fest und sehen darin einen Grund für ihre seltenere Autonutzung. Choo und Mokhtarian (2004: 211 f.) finden anhand der Nutzung bestimmter Autotypen geschlechtsspezifische Unterschiede im Umweltbewusstsein und im Spaß am Autofahren. In Götz' (2011: 339 f.) einstellungsbasierten Mobilitätstypen sind „traditionell Naturorientierte" mit hohem Fußwegeanteil und „Traditionell-Häusliche“ mit gehobenem Sicherheitsbedürfnis überwiegend Frauen, ,risikoorientierte Autofahrer" hingegen zu 90 Prozent Männer. Ein weiterer Grund für die seltenere Autonutzung von Frauen liegt nach Matthies, Kuhn und Klöckner (2002: 171) in der schwächeren Gewohnheitsprägung ihrer Alltagsmobilität. Brennecke (1994: 7) sieht jedoch in der stärkeren Verantwortung bzw. Zuständigkeit für die Kinder eine gröBere Umweltverantwortung bei den Frauen, was den Bezug zwischen Präferenz- und Rollentheorie verdeutlicht.

Rollentheoretische Ansätze verstehen die geschlechtsspezifische Arbeitsteilung als zentralen Faktor von Geschlechterrollen und diese wiederum als Grund für geschlechtsspezifische Mobilität. So argumentiert Dobbs (2005: 272 ff.), dass Frauen wegen ihrer komplexen Aktivitätsmuster auch bei guter ÖPNV-Anbindung für ihre spezifischen Mobilitätsbedürfnisse bevorzugt das Auto nutzen. Zeitliche sowie räumliche Flexibilität, Zuverlässigkeit und Zeitersparnis sind hierbei zentral. Dobbs (2005: $272 \mathrm{ff}$.) sieht das Auto sozusagen als Mittel zur Vereinbarkeit von 
Erwerbs- und Versorgungsarbeit. Best und Lanzendorf (2005: 120) erkennen Ähnliches für berufstätige Mütter mit deren Doppelbelastung. Als Kehrseite des Autos sieht Buhr (1999: 126), dass die steigende Autoverfügbarkeit und -nutzung von Müttern beispielsweise die Verpflichtung zu Bring- und Holdiensten erhöht. Dagegen steht der ÖPNV dem Auto in der Bewältigung komplexer Alltagsmuster und Wegeketten, Flexibilität, Komfort, Zeitaufwand und Zuverlässigkeit nach (vgl. Hamilton/Jenkins 2000; Hensher/ Reyes 2000: 357; Dobbs 2005: 277). Diese Begründung gilt vorbehaltlich einer entsprechenden Pkw-Verfügbarkeit der Frauen, was den Zusammenhang zwischen Rollen- und Restriktionstheorie zeigt.

Restriktionstheoretisch argumentieren Rutherford und Wekerle (1988). Frauen sind demnach häufiger captive riders, die in Folge ihrer geringen Pkw-Verfügbarkeit auf den ÖPNV angewiesen sind (ähnlich auch Best/Lanzendorf 2005: 115), während Männer als choice riders des ÖPNV wahlfrei sind und auch andere Alternativen - nämlich das Auto - hätten. Eine Restriktion kann auch das Sicherheitsbedürfnis darstellen. Dobbs (2005: 274) meint hingegen, der ÖPNV entspräche nicht den Sicherheitsbedürfnissen von Frauen, insbesondere bei Dunkelheit. Hagman (2003: 3 f.) führte hierzu Interviews mit schwedischen Autofahrerinnen/Autofahrern: In erster Linie bewerteten Frauen die Sicherheit als spezifischen Vorteil des Autos. Indem sich geschlechtsspezifische Sicherheitsbedürfnisse restriktiv auf das Verkehrsverhalten auswirken können, wird die Verknüpfung zwischen Restriktions- und Präferenztheorie offensichtlich.

Bezogen auf die Pkw-Verfügbarkeit und Verteilung innerhalb eines Haushaltes zwischen Mann und Frau erklärt die rational-ökonomische Theorie die seltenere Pkw-Nutzung von Frauen. Eine häufigere Pkw-Nutzung der Männer stellt hiernach in Haushalten mit mehr Führerscheinbesitzern als Pkw eine rationale Haushaltsentscheidung dar: Wegen des höheren Einkommens bringt eine Zeitersparnis des Mannes durch die Pkw-Nutzung dem Haushalt einen größeren Nutzen, als wenn die geringer verdienende Frau mittels des Autos Zeit spart (vgl. Bamberg 1996: 14). In diesem theoretischen Ansatz drückt sich also eine ökonomische Haushaltslogik aus, die der individuellen Logik übergeordnet ist. Hier ergeben sich insbesondere Anknüpfungspunkte zur Restriktions- sowie zur Rollentheorie.

Alle genannten theoretischen Ansätze mit Ausnahme der Präferenztheorie argumentieren strukturalistisch in dem Sinne, dass sie individuelles Handeln als abhängig von übergeordneten Strukturen und Restriktionen begreifen. Damit können sie Einschränkungen der Mobilität (von Frauen) erklären, stehen aber tendenziell im Widerspruch zu Zeitdiagnosen, die die zunehmende Freiheit individueller Lebensstile und individuellen Handelns betonen und dabei auch den Wandel des Geschlechterverhältnisses der letzten
Jahrzehnte hervorheben (z. B. Beck 1986). Auffallend ist darüber hinaus, dass die unterschiedlichen theoretischen Ansätze in der Forschung kaum systematisch als miteinander konkurrierende Hypothesen geprüft, sondern eher argumentativ nebeneinander gestellt werden.

Vor dem Hintergrund des genannten Wandels und den bislang fehlenden längerfristigen, differenzierten Trendanalysen der geschlechtsspezifischen Alltagsmobilität in Deutschland stellt sich die erste Forschungsfrage dieses Beitrags: Wie hat sich die Pkw-Nutzung als Fahrerin/Fahrer von Männern und Frauen in Deutschland langfristig entwickelt? Im Hinblick auf die verschiedenen Erklärungsansätze für geschlechtsspezifisches Verkehrsverhalten ergibt sich die nächste Frage zu den geschlechtsspezifischen Unterschieden hinsichtlich der Pkw-Nutzung: Resultieren sie aus sozialen Rollen (Erwerbstätigkeit, Haushaltskontext), aus Restriktionen (geringere Pkw-Verfügbarkeit der Frauen), aus rational-ökonomischen Haushaltsentscheidungen über eine knappe Ressource Pkw oder aus unterschiedlichen Normen und Präferenzen? Letztere werden dabei mangels Daten nur indirekt aus den Ergebnissen erschlossen, während die anderen Erklärungsansätze direkt überprüft werden.

\section{Daten und Methodik}

Als Datenbasis werden die deutschen Verkehrserhebungen KONTIV (Kontinuierliche Erhebung zum Verkehrsverhalten; 1976, 1982 und 1989) und KONTIV-Nachfolgeerhebungen MiD (Mobilität in Deutschland; 2002 und 2008) verwendet. Die fünf Erhebungen sind mit einigen Einschränkungen als Zeitreihe verwendbar, allerdings mit deutlichem Aufbereitungs- und Vereinheitlichungsbedarf aufgrund methodischer Variationen, unterschiedlicher Grundgesamtheiten und Befragungsinhalte. Zugunsten einer möglichst guten Vergleichbarkeit werden zum einen zahlreiche Variablen zwischen den fünf Datensätzen auf den kleinsten gemeinsamen Nenner vereinheitlicht. Zum anderen wird die Grundgesamtheit auf volljährige Personen deutscher Nationalität aus den alten Bundesländern begrenzt. Die Datenaufbereitung ist im Detail beschrieben in Konrad (2016). Den Schwächen dieser Daten steht ihre Einzigartigkeit (für Deutschland bezogen auf die frühere Bundesrepublik) als lange Zeitreihe gegenüber, während das Deutsche Mobilitätspanel erst seit 1994 erhoben wird.

Die Pkw-Nutzung wird wie folgt operationalisiert: Es wird für die am Stichtag mobilen Personen (mit Fahrerlaubnis) untersucht, ob eine Person am Stichtag mindestens einmal als Autofahrerin/Autofahrer unterwegs war. Nach einer einleitenden deskriptiven Auswertung wird mittels binärlogistischer Regressionen die Wahrscheinlichkeit der Autonutzung in Abhängigkeit einer Vielzahl von Faktoren ge- 
schätzt. Für jedes der fünf Erhebungsjahre wird ein Regressionsmodell angenommen, die fünf identisch strukturierten Modelle werden als Reihe nebeneinander gestellt.

In den Modellen werden für alle Merkmale auch deren Interaktionen mit dem Geschlecht kontrolliert, um möglichst differenziert Trends für Männer und Frauen nachzuzeichnen. Aus dem Stand der Forschung ergeben sich folgende relevante Merkmale, die durchweg als DummyVariablen kontrolliert werden:

- Geschlecht,

- Tätigkeit (Vollzeit, Teilzeit, in Ausbildung, nicht erwerbstätig),

- Personentyp/Haushaltskontext (alleinlebend, Paar mit Kleinkind/Kleinkindern, Paar mit Schulkind/Schulkindern aber ohne Kleinkinder, Paar ohne Kind, alleinerziehend, Erwachsenenhaushalt, erwachsenes Kind, Großeltern),

- Gemeindegrößenklasse $(<5000,5000$ bis $<20.000$, 20.000 bis $<100.000,100.000$ bis $<500.000, \geq 500.000$ Einwohner),

- Höchster Schulabschluss,

- Altersgruppe in Jahren (12 Altersgruppen).

Der Personentyp bzw. Haushaltskontext bezeichnet die Position einer Person im Gefüge des Haushalts, in dem sie lebt. Aus den Alters- und Geschlechtsangaben aller Mitglieder eines Haushaltes wurde die Haushaltsform rekonstruiert (z. B. Single-Haushalt, Paar ohne Kinder, Paar mit Kind). Aus den personenbezogenen Angaben zu Alter und Geschlecht wurde dann der Personentyp definiert, also ob es sich beispielsweise in einer Familie um die Mutter, den Vater, ein erwachsenes oder ein minderjähriges Kind handelt. Dabei ist eine gewisse Unschärfe unvermeidbar. Ob es sich um ein Eltern-Kind-Verhältnis handelt, wurde an einem Altersunterschied von mindestens 18 Jahren festgemacht, geringere Altersunterschiede wurden bei zwei erwachsenen Personen unterschiedlichen Geschlechts als Indikator für eine Paar-Beziehung gewertet (im Detail hierzu vgl. Konrad 2016). Familien werden nach dem Alter des jüngsten Kindes (unter sechs Jahre oder mindestens sechs Jahre) unterschieden, Elternpaare mit ausschließlich erwachsenen Kindern im Haushalt werden als reine Erwachsenen-Haushalte definiert, erwachsene Kinder leben mit ihren Eltern und minderjährigen Geschwistern zusammen, Großeltern sind Personen aus 3-Generationen-Haushalten und leben mit zwei jüngeren Generationen zusammen.

Altersgruppen werden mit einer Spannweite von jeweils 6,5 Jahren gebildet. Der Hintergrund für diese „schiefe“ Einteilung ist, dass die Erhebungen über den gesamten Zeitraum jeweils sechs bzw. sieben Jahre im Wechsel auseinander liegen (mit einer fehlenden Erhebung 1995). Dabei werden jeweils die Befragten jedes sechsten bzw. siebten Geburtsjahrganges zufällig zu gleichen Teilen den beiden angrenzenden Altersgruppen zugeordnet.
Auf der Basis des Forschungsstandes nehmen wir für die Nutzung und Verteilung des/der Autos zwischen Männern und Frauen deutliche Unterschiede in Abhängigkeit von der Pkw-Ausstattung von Haushalten als möglicher Restriktion an. Daher werden die Analysen parallel für vollmotorisierte (Anzahl Pkw $\geq$ Anzahl Fahrberechtigte) und teilmotorisierte $(0<$ Anzahl Pkw < Anzahl Fahrberechtigte) Haushalte durchgeführt.

Durch diese Aufgliederung der Analyse werden Restriktionen als Determinanten der Pkw-Nutzung ausgeklammert, da hinsichtlich der Pkw-Verfügbarkeit zumindest theoretisch die Männer und Frauen dieselben Möglichkeiten zur Nutzung eines Pkw haben (Teil- bzw. Vollmotorisierung). Das Vorgehen erlaubt eine Einschätzung, inwiefern rational-ökonomisch orientierte Aushandlungen bzw. Entscheidungen in Haushalten über die Verteilung der knappen Ressource Pkw zu einer geschlechtsspezifischen Pkw-Nutzung beitragen. Ist das der Fall, sollte der Unterschied zwischen Männern und Frauen in teilmotorisierten Haushalten größer als in vollmotorisierten Haushalten sein. Die Erklärungskraft der Rollentheorie kann zum einen über die Kontrolle der Erwerbstätigkeit abgeschätzt werden. So sollte rollentheoretisch gesehen der gleiche Erwerbsumfang von Männern und Frauen (z. B. Vollzeit) in der gleichen Pkw-Nutzung münden. Zum anderen ist der Haushaltskontext ein Indikator für familiäre Rollenverpflichtungen und besonders die geschlechtsspezifische Arbeitsteilung. Demnach sollten erwerbstätige Mütter als Gruppe mit komplexen Aktivitätsmustern sich von anderen Frauen durch eine häufige Autonutzung abheben. Die Relevanz geschlechtsspezifischer Präferenzen und Gewohnheitsprägungen für die Pkw-Nutzung kann aufgrund fehlender einstellungsbezogener Angaben nicht direkt empirisch überprüft werden, dennoch sind näherungsweise Schlussfolgerungen hierzu möglich. Hierauf würden nennenswerte Geschlechterunterschiede selbst bei Vollmotorisierung und gleichem Erwerbsumfang (als Kontrolle der Restriktions- und Rollentheorie) hinweisen. Dagegen würde eine stärkere Pkw-Nutzung des Mannes bei Teilmotorisierung (ein Pkw für zwei Partner), aber gleichem Erwerbsumfang ein haushaltsbezogenes rational-ökonomisches Kalkül nahelegen oder auf eine stärkere Durchsetzungsfähigkeit des Mannes oder einen Verzicht der Frau hinweisen.

Die als Einflussgrößen der Pkw-Nutzung in die Modelle integrierten Merkmale bilden nicht das vollständige Set relevanter Merkmale ab. Weitere Einflussfaktoren wie das individuelle Einkommen können in diesem Beitrag nicht berücksichtigt werden, da sie in der verwendeten Datenbasis nicht vorliegen. Wie bereits erwähnt gilt dasselbe für individuelle Einstellungen, Normen und Präferenzen. Nichtsdestotrotz bilden die hier betrachteten Einflussgrößen die wesentlichen Rahmenbedingungen des Verkehrsverhaltens 
im Geschlechterverhältnis und diesbezüglicher Trends ab, wie sie sich aus dem Stand der Forschung ergeben.

Für die Analysen werden die Daten nach Haushaltsgröße, Alter, Regionstyp, Wochentag und Monat gewichtet, um die Stichprobenzusammensetzung an die Bevölkerungszusammensetzung des jeweiligen Erhebungsjahres anzupassen und saisonale Verzerrungen auszugleichen. Die Vielzahl statistischer Tests erhöht die Wahrscheinlichkeit, fälschlicherweise zufällige Signifikanzen anzunehmen; dieses Phänomen ist als Alphafehler-Kumulierung bekannt (Schendera 2008: 26). Zum Ausgleich dessen wird eine Bonferroni-Korrektur zur Alphafehler-Adjustierung vorgenommen: Alpha (hier 5 Prozent) wird durch die Anzahl der Tests je Modell dividiert und als korrigiertes Signifikanzniveau zugrunde gelegt (vgl. Bland/Altman 1995: 170). Dies führt zu einer im Vergleich zum gängigen Vorgehen (ohne Adjustierung) deutlich strengeren Interpretation der Signifikanz.

Die Zeitreihen aus je fünf Regressionsmodellen mit zahlreichen Interaktionstermen (ersten bis dritten Grades) weisen eine Komplexität auf, die eine Interpretation von Koeffizienten und das Ablesen zeitlicher Verläufe erschwert. Daher und um Fehlinterpretationen zu vermeiden, werden aus den Regressionsmodellen anhand der Regressionsgleichung Wahrscheinlichkeiten für ausgewählte Personengruppen (Merkmalskonstellationen) berechnet und als Trendlinien grafisch dargestellt. Die Basisgruppe dieser Berechnungen und damit die Vergleichsgruppe ist definiert als die Kombination der jeweils häufigsten Merkmalsausprägungen je Erklärungsgröße: Vollzeiterwerbstätige (= häufigste Tätigkeit), 44-49,5 Jahre alt (= häufigste Altersgruppe), höchster Schulabschluss niedriger als Abitur (= häufigster Schulabschluss), Paar ohne Kind (= häufigster Haushaltskontext), Wohnort zwischen 5000 und unter 20.000 Einwohnern (= häufigste Gemeindegrößenklasse). Die Basisgruppe als eine theoretische Gruppe (ähnlich der Modell-Konstanten als Kombination aller Merkmals-Ausprägungen $=0$ ) wurde in dieser Weise definiert, um durch eine starke Besetzung der einzelnen Parameter eine statistisch belastbare Vergleichsbasis für ergänzende, variierende Beispielrechnungen zu haben. Die Beispielrechnungen erfolgen zudem für ausgewählte Variationen der Basisgruppe, nämlich Teilgruppen mit besonders großen oder geringen Verhaltensunterschieden zwischen Männern und Frauen oder besonders starken oder schwachen Angleichungstrends. Dabei variiert immer nur ein Merkmal gegenüber der Basisgruppe. In den Beispielrechnungen können sowohl geschlechtsspezifische Unterschiede in der Pkw-Nutzung unabhängig von anderen genderrelevanten Merkmalen wie der Erwerbstätigkeit oder Pkw-Verfügbarkeit als auch Trends veranschaulicht werden, die über die steigende Führerschein- und Pkw-Ausstattung hinausgehen. Die Beschreibung der Ergebnisse stützt sich der Anschaulichkeit halber vor allem auf die Trenddiagramme. Aussagen zu Signifikanzen beziehen sich auf die Regressionsanalysen (vgl. Tab. 2 und 3).

In den Regressionsmodellen wurden zahlreiche nicht signifikante Faktoren beibehalten, da sie aus der Literaturrecherche als genderrelevant hervorgehen. Die Kontrolle der größtmöglichen Bandbreite genderrelevanter Faktoren erlaubt eine Eingrenzung des Erklärungsgehalts der zuvor beschriebenen Theorien. Auch bei fehlender Signifikanz könnten einige Faktoren die Ausprägung des Unterschieds zwischen Männern und Frauen beeinflussen und bei Nichtbeachtung im Modell zu Unschärfen auch bei den Beispielrechnungen führen. Die Beibehaltung von Faktoren, die in einzelnen Regressionen nicht signifikant sind, und die daraus resultierende Einheitlichkeit der Modelle innerhalb und zwischen den beiden Modellzeitreihen erlaubt zum einen die Abbildung von Trends im Zeitverlauf für fest definierte Personengruppen und zum anderen den Vergleich zwischen den beiden betrachteten Teilgruppen (teil- und vollmotorisiert).

Die KONTIV 1989 weist aufgrund erhebungsmethodischer Abweichungen von den anderen Erhebungen eine schlechte Datenqualität auf (für Einzelheiten vgl. Konrad 2016) und weicht von Trends häufig ab. Daher werden die Werte für 1989 in den Trenddiagrammen ausgespart. 1995 fand keine Erhebung statt. Um äquidistante Abstände in den Trenddiagrammen zu erhalten, werden die Trendlinien zwischen 1982 und 2002 verbunden. Zur Verdeutlichung werden für die Zeitpunkte 1989 und 1995 in den Diagrammen keine Datenpunkte dargestellt.

\section{Auswertung und Ergebnisse}

Wir beginnen mit einer Beschreibung der Entwicklung der Pkw-Nutzung von Männern und Frauen. Die Prozentangaben in Tab. 1 zeigen die Anteile der volljährigen deutschen Männer und Frauen aus den alten Bundesländern, die am Stichtag mindestens einmal Auto gefahren sind. Die Auswertung bestätigt das im aktuellen Stand der Forschung festgestellte Aufholen der Frauen, das im Zusammenhang mit der steigenden Motorisierung gesehen wird. Ein etwa konstanter Anteil bei den Männern steht einer sich mehr als verdoppelnden Autonutzug durch Frauen gegenüber, dennoch besteht auch 2008 noch ein nennenswerter und signifikanter Unterschied.

In den folgenden Regressionsanalysen werden die geschlechtsspezifischen Unterschiede und der Trend in den komplexen Kontext von Faktoren eingeordnet, die die Verkehrsmittelnutzung beeinflussen und die sich selbst zwischen 1976 und 2008 verändern. Dazu gehören beispielsweise der Führerscheinbesitz (Anstieg bei den Frauen von 39 auf 86 Prozent, bei den Männern von 76 auf 94 Prozent), die (uneingeschränkte) Pkw-Verfügbarkeit (Anstieg bei den 
Tab. 1 Entwicklung der Pkw-Nutzung als Fahrerin/Fahrer bei Männern und Frauen

\begin{tabular}{lllll}
\hline Jahr & Männer (in \%) & Frauen (in \%) & Ratio & Signifikanz \\
\hline 1976 & 63,1 & 22,0 & 2,87 & $*$ \\
1982 & 63,4 & 30,4 & 2,08 & $*$ \\
1989 & 63,4 & 29,7 & 2,14 & $*$ \\
1995 & Keine Erhebung & & & $*$ \\
2002 & 68,0 & 51,8 & 1,31 & $*$ \\
2008 & 66,0 & 51,0 & 1,29 & $*$ \\
\hline
\end{tabular}

Berechnung nach KONTIV/MiD 1976-2008; volljährige, am Stichtag mobile Personen, deutsch, alte Bundesländer ${ }^{*} p \leq 0,01, \mathrm{Chi}^{2}$ nach Pearson

Frauen von 36 auf 81 Prozent, bei den Männern schwankend zwischen 86 und 90 Prozent) oder die Erwerbstätigkeit (Anstieg bei den Frauen von 43 auf 64 Prozent ungeachtet des Erwerbsumfangs, bei den Männern schwankend zwischen 76 und 83 Prozent).

In der jeweiligen vollmotorisierten und teilmotorisierten Basisgruppe entwickelt sich die Pkw-Nutzung im Geschlechterverhältnis sichtlich unterschiedlich (vgl. Abb. 1 und 2). 1976 ist die Pkw-Nutzung der Männer unabhängig von der Pkw-Ausstattung des Haushalts hoch, bei den Frauen wird sie durch eine Teilmotorisierung des Haushalts erheblich eingeschränkt. Die Männer haben demnach bezüglich der knappen Ressource Pkw die first choice, Aushandlungsprozesse gehen zu ihren Gunsten aus (Abb. 2). Doch selbst bei Vollmotorisierung (vgl. Abb. 1) fahren Frauen seltener Auto, trotz ständiger Pkw-Verfügbarkeit und auch ansonsten ähnlicher Rahmenbedingungen (z. B. Vollzeiterwerbstätigkeit). Allerdings verschiebt sich dieses Verhältnis bis 2008 deutlich: In den vollmotorisierten Haushalten nutzen die Frauen den ihnen zur Verfügung stehenden Pkw immer häufiger, der Unterschied zu ihren männlichen Pendants wird minimal, aber bleibt signifikant (signifikanter negativer Effekt „MehrpersonenHaushalt*weiblich" in Tab. 2). In den teilmotorisierten Haushalten (vgl. Abb. 2) verlagert sich die relative Verteilung des Pkw deutlich von den Männern zu den Frauen. Bis 2008 bleibt auch hier die Pkw-Nutzung der Frauen signifikant niedriger als die der Männer (signifikanter negativer Effekt „weiblich“ in Tab. 3). Inwiefern es sich bei den vom sonst klaren Trend abweichenden Werten in 2008 um Ausreißer oder eine Trendveränderung handelt, ist anhand nachfolgender Erhebungen zu prüfen.

Die Tätigkeit bzw. der Erwerbsumfang ist klar geschlechtsspezifisch und eine zentrale Kenngröße in der Diskussion um geschlechtsspezifische Mobilität. Sie steht in dieser Analyse als Indikator für die geschlechtsspezifische Rollenverteilung und damit zusammenhängende Mobilitätsunterschiede zwischen Männern und Frauen.

In teil- und vollmotorisierten Haushalten fahren nicht erwerbstätige Personen seltener Auto als Vollzeiterwerbstätige (vgl. Abb. 3 und 4; zum Teil signifikanter Effekt „nicht erwerbstätig“ in Tab. 2 und 3). In teilmotorisierten
Haushalten gilt das verstärkt für die Männer, zumindest bis 2002 (positiver, nicht signifikanter Effekt „,nicht erwerbstätig*weiblich“ schwächt negativen Effekt ,nicht erwerbstätig" für Frauen ab; Tab. 3). Nicht erwerbstätige Frauen, vielfach Hausfrauen, übernehmen viele Versorgungsaufgaben und haben dafür vermutlich einen vergleichsweise hohen Pkw-Bedarf. Eine ergänzende Auswertung des Autofahrens auf verschiedenen Wegezwecken zeigt, dass zum Bringen und Holen von Personen mit Abstand das Auto dominiert und häufiger das Auto genutzt wird als auf allen anderen Wegezwecken (vgl. Konrad 2016: 184).

Im Zeitverlauf reduzieren in teilmotorisierten Haushalten speziell die vollzeiterwerbstätigen Männer ihre Autonutzung (vgl. Abb. 4; abnehmender Effekt „nicht erwerbstätig“ in Tab. 3) zugunsten ihrer Partnerinnen. Vor allem diejenigen Männer reduzieren also ihre Pkw-Nutzung, bei denen das am ehesten eine Herausforderung für ihre Alltagsorganisation bedeutet. Interpretiert werden kann dies als Umdenken in Richtung einer egalitären Ressourcenverteilung.

Der Haushaltskontext beeinflusst sowohl in vollmotorisierten als auch in teilmotorisierten Haushalten nur die Pkw-Nutzung der Frauen, während die Männer den Pkw fast unbeeinflusst vom Haushaltskontext nutzen. In vollmotorisierten Haushalten (vgl. Abb. 5) zeigt sich die seltenere Pkw-Nutzung der Frauen, wie sie für die Basisgruppe festzustellen war, nur in Mehrpersonenhaushalten, mit und ohne Kinder (signifikanter negativer Effekt „Mehrpersonenhaushalt*weiblich“ in Tab. 2). Zwischen alleinlebenden Männern und Frauen mit Pkw (vgl. Abb. 5) unterscheidet sich die Pkw-Nutzung nicht (in Tab. 2 ,weiblich“ nicht signifikant). Prinzipiell dürften Haushalte dann vollmotorisiert sein, wenn ein entsprechender Bedarf besteht. Trotzdem fahren Frauen in allen vollmotorisierten Mehrpersonenhaushalten und zu jedem Erhebungszeitpunkt seltener als ihre Partner (und als alleinlebende Frauen) Auto. Eine Erklärung dürften gemeinsam mit dem Partner als Beifahrerin zurückgelegte Wege sein, wobei sich die Frage nach dem Grund für die Verteilung Fahrerin/Fahrer - Beifahrerin/ Beifahrer auf gemeinsamen Wegen stellt. Möglicherweise sind die Pkw-Routinen bei Frauen aber auch generell nicht so stark ausgeprägt. Scheinbar besteht zwar ein genereller 


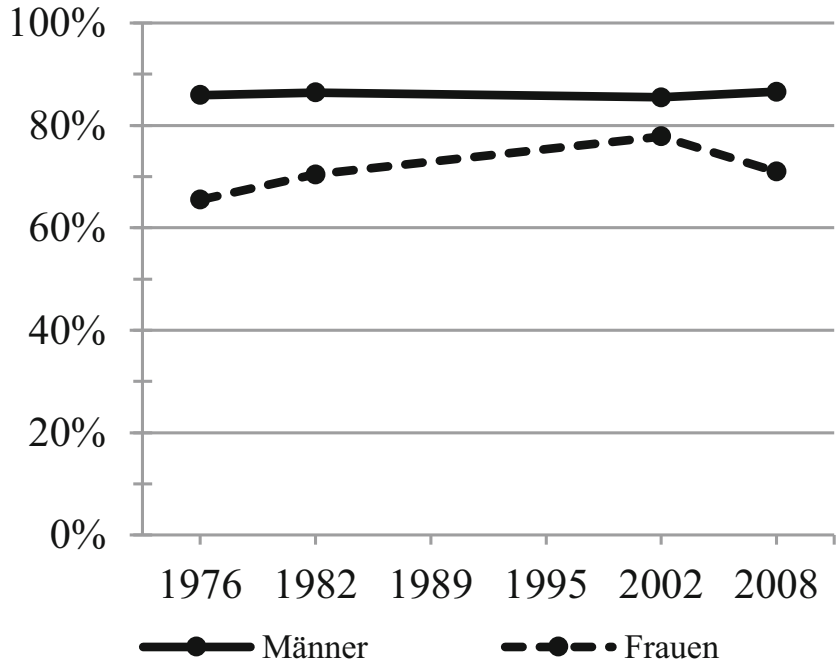

Abb. 1 Entwicklung der Pkw-Nutzung als Fahrerin/Fahrer in der vollmotorisierten Basisgruppe (Basisgruppe: Vollzeiterwerbstätig, 44-49,5 Jahre, ohne Abitur, Paar ohne Kind, Wohnort 5000 bis $<20.000$ Einwohner, Vollmotorisiert: mindestens so viele Pkw wie fahrfähige Personen im Haushalt)

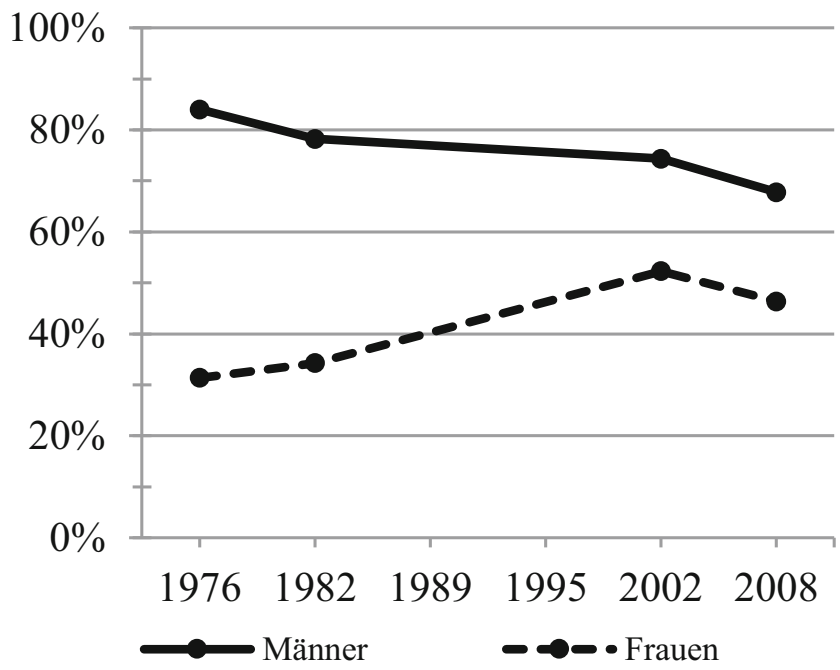

Abb. 2 Entwicklung der Pkw-Nutzung als Fahrerin/Fahrer in der teilmotorisierten Basisgruppe (Basisgruppe: Vollzeiterwerbstätig, 44-49,5 Jahre, ohne Abitur, Paar ohne Kind, Wohnort 5000 bis $<20.000$ Einwohner, Teilmotorisiert: weniger Pkw als fahrfähige Personen im Haushalt)

Bedarf nach einem Zweit- oder Drittwagen, der sich aber nur begrenzt in einer Nutzung dieses Autos niederschlägt.

In teilmotorisierten Haushalten (vgl. Abb. 6) sind geschlechtsspezifische Unterschiede der Pkw-Nutzung deutlich größer als in vollmotorisierten Haushalten (vgl. Abb. 5). Dies dürfte das Ergebnis rational-ökonomisch orientierter Haushaltsentscheidungen über die knappe Ressource Pkw und/oder Ausdruck ungleicher Verhandlungspositionen von Männern und Frauen sein. Für ungleiche Verhandlungspositionen spricht, dass Mütter in teilmo-

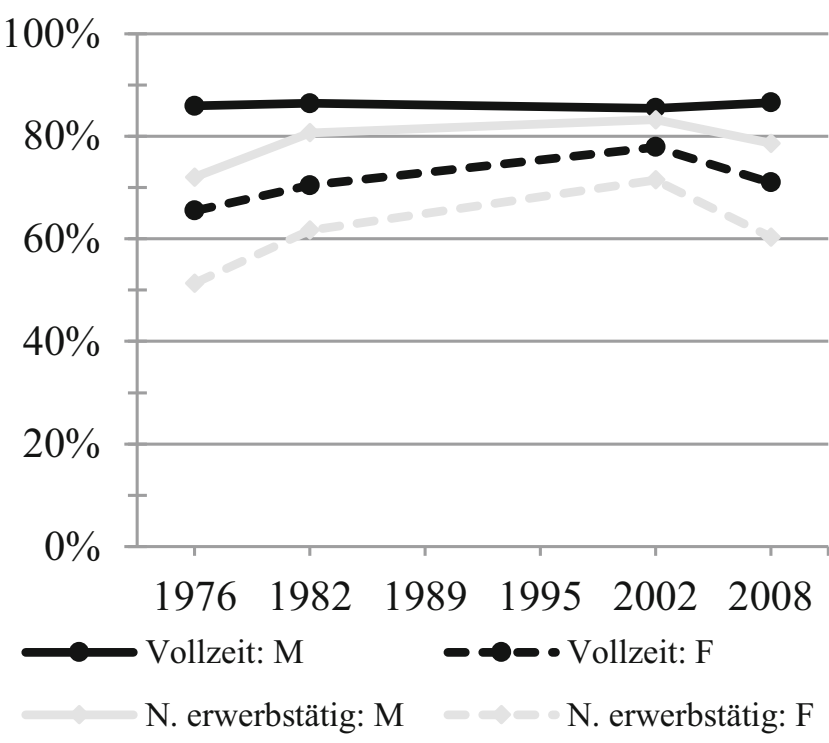

Abb. 3 Pkw-Nutzung als Fahrerin/Fahrer in vollmotorisierten Haushalten differenziert nach Tätigkeit (Gruppen: vollzeiterwerbstätig/nicht erwerbstätig, 44-49,5 Jahre, ohne Abitur, Paar ohne Kind, Wohnort 5000 bis $<20.000$ Einwohner, Vollmotorisiert: mindestens so viele Pkw wie fahrfähige Personen im Haushalt)

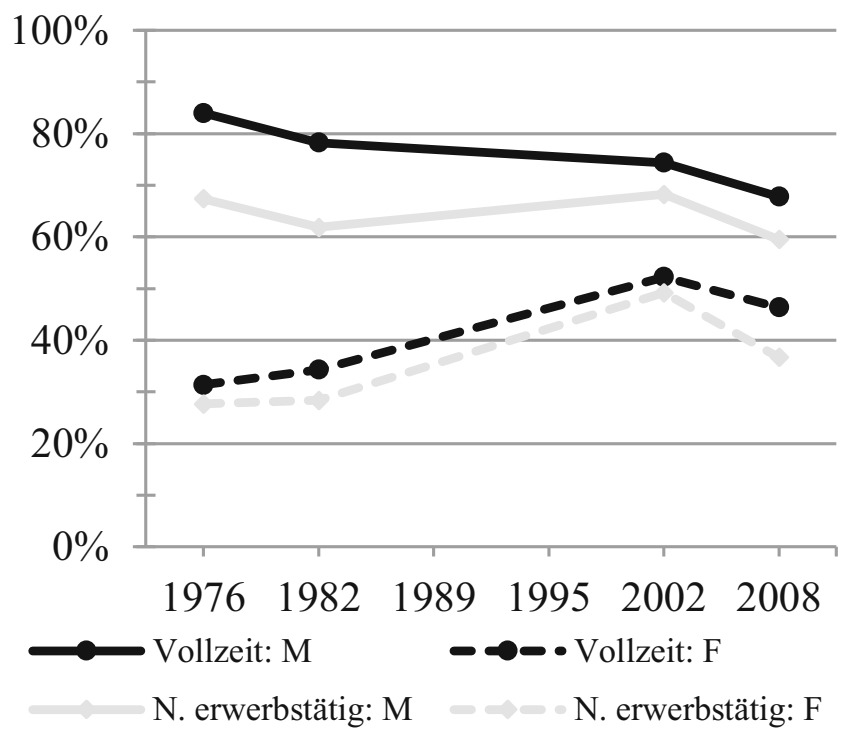

Abb. 4 Pkw-Nutzung als Fahrerin/Fahrer in teilmotorisierten Haushalten differenziert nach Tätigkeit (Gruppen: vollzeiterwerbstätig/nicht erwerbstätig, 44-49,5 Jahre, ohne Abitur, Paar ohne Kind, Wohnort 5000 bis $<20.000$ Einwohner, Teilmotorisiert: weniger Pkw als fahrfähige Personen im Haushalt)

torisierten Haushalten im Vergleich zu Frauen aus Paaren ohne Kind eine „bessere Verhandlungsposition“ oder einen höheren „Nutzungsdruck“ haben, der ihre häufigeren Autofahrten begründet - was ihnen auch gelingt (vgl. Abb. 6; zum Teil signifikante positive Interaktion „Paar mit Kind*weiblich“ in Tab. 3). Allerdings fahren Väter im Gegenzug nicht seltener Auto. Demnach wird die knappe 
Tab. 2 Logit-Regression der Pkw-Nutzung als Fahrerin/Fahrer in vollmotorisierten Haushalten. (Eigene Berechnung nach KONTIV/MiD 1976-2008)

\begin{tabular}{|c|c|c|c|c|c|c|c|c|c|c|}
\hline & 1976 & & 1982 & & 1989 & & 2002 & & 2008 & \\
\hline & $\mathrm{B}$ & Sign. & $\mathrm{B}$ & Sign. & B & Sign. & $\mathrm{B}$ & Sign. & $\mathrm{B}$ & Sign. \\
\hline Konstante & 2,743 & * & 1,539 & $*$ & 1,441 & $*$ & 1,666 & * & 1,760 & * \\
\hline \multicolumn{11}{|l|}{ Geschlecht, Referenz: männlich } \\
\hline Weiblich & $-1,809$ & $*$ & $-0,105$ & & $-0,233$ & & 0,175 & & $-0,237$ & \\
\hline \multicolumn{11}{|c|}{ Altersklasse, Referenz: 44-49,5 Jahre } \\
\hline $18-23,5$ & 0,328 & & $-0,147$ & & 0,365 & & 0,201 & & $-0,044$ & \\
\hline $23.5-30$ & 0,136 & & 0,102 & & 0,154 & & 0,024 & & $-0,248$ & \\
\hline $31-36,5$ & 0,497 & & 0,119 & & 0,256 & & $-0,149$ & & $-0,015$ & \\
\hline $36,5-43$ & 0,016 & & $-0,023$ & & 0,162 & & $-0,207$ & & $-0,148$ & \\
\hline \multicolumn{11}{|l|}{ (44-49,5 Referenz) } \\
\hline $49,5-56$ & $-0,129$ & & $-0,167$ & & 0,152 & & $-0,038$ & & $-0,166$ & \\
\hline $57-62,5$ & $-0,093$ & & $-0,315$ & & 0,185 & & $-0,081$ & & $-0,123$ & \\
\hline $62,5-69$ & $-0,388$ & & $-0,308$ & & 0,034 & & $-0,588$ & ${ }^{*}$ & $-0,093$ & \\
\hline $70-75,5$ & $-0,849$ & & $-0,510$ & & $-0,082$ & & $-0,359$ & & $-0,449$ & \\
\hline $75,5-82$ & $-0,743$ & & $-1,027$ & & $-0,139$ & & $-0,857$ & * & $-0,315$ & \\
\hline $83-88,5$ & 19,751 & & $-0,777$ & & $-0,554$ & & $-0,788$ & & $-0,569$ & \\
\hline $\mathrm{Ab} 88,5$ & 19,776 & & $-22,632$ & & & & $-22,159$ & & $-22,166$ & \\
\hline $18-23,5^{*}$ weiblich & $-0,123$ & & 0,778 & & 0,050 & & 0,004 & & 0,332 & \\
\hline $23,5-30^{*}$ weiblich & 0,268 & & 0,055 & & $-0,138$ & & 0,462 & & 0,329 & \\
\hline $31-36,5^{*}$ weiblich & $-0,085$ & & 0,174 & & $-0,280$ & & 0,196 & & $-0,101$ & \\
\hline $36,5-43^{*}$ weiblich & 0,475 & & 0,397 & & $-0,026$ & & 0,207 & & 0,027 & \\
\hline \multicolumn{11}{|l|}{ (44-49,5 Referenz*weiblich) } \\
\hline $49,5-56^{*}$ weiblich & 0,754 & & 0,035 & & $-0,485$ & & $-0,245$ & & 0,261 & \\
\hline $57-62,5^{*}$ weiblich & 0,208 & & 0,244 & & $-0,290$ & & $-0,204$ & & 0,333 & \\
\hline $62,5-69^{*}$ weiblich & 0,823 & & $-0,088$ & & 0,021 & & 0,056 & & 0,066 & \\
\hline $70-75,5^{*}$ weiblich & 0,153 & & $-0,154$ & & $-0,170$ & & $-0,449$ & & $-0,077$ & \\
\hline $75,5-82^{*}$ weiblich & 21,288 & & 2,137 & & $-0,141$ & & $-0,340$ & & 0,216 & \\
\hline $83-88,5^{*}$ weiblich & & & 20,942 & & 20,840 & & 1,127 & & $-0,853$ & \\
\hline \multicolumn{11}{|l|}{$\mathrm{Ab} 88,5^{*}$ weiblich } \\
\hline \multicolumn{11}{|c|}{ Tätigkeit, Referenz: Vollzeiterwerbstätige } \\
\hline Nicht erwerbstätig & $-0,861$ & $*$ & $-0,422$ & & $-0,784$ & * & $-0,169$ & & $-0,559$ & * \\
\hline Nicht erwerbstätig*weiblich & 0,274 & & 0,031 & & 0,273 & & $-0,170$ & & 0,085 & \\
\hline Teilzeit & $-0,076$ & & $-0,514$ & & $-0,465$ & & $-0,228$ & & $-0,128$ & \\
\hline Teilzeit ${ }^{*}$ weiblich & $-0,213$ & & 0,445 & & 0,310 & & 0,106 & & $-0,004$ & \\
\hline Ausbildung & $-0,460$ & & $-0,447$ & & $-0,704$ & $*$ & $-0,380$ & & $-0,699$ & * \\
\hline Ausbildung*weiblich & 1,617 & & $-0,077$ & & 0,072 & & 0,000 & & 0,220 & \\
\hline \multicolumn{11}{|c|}{ Höchster Schulabschluss (für Personen nicht mehr in Ausbildung), Referenz: niedriger als Abitur } \\
\hline Abitur & 0,264 & & 0,277 & & 0,054 & & $-0,060$ & & 0,042 & \\
\hline Abitur*weiblich & 0,109 & & 0,084 & & $-0,177$ & & 0,155 & & 0,102 & \\
\hline \multicolumn{11}{|c|}{ Personentyp/Haushaltskontext, Referenz: Alleinlebende } \\
\hline Mehrpersonenhaushalt & $-0,456$ & & 0,260 & & 0,267 & & 0,005 & & 0,230 & \\
\hline Mehrpersonenhaushalt* weiblich & 0,353 & & $-1,125$ & * & $-0,684$ & $*$ & $-0,516$ & * & $-0,654$ & * \\
\hline Paar mit Kind & $-0,044$ & & 0,134 & & $-0,178$ & & 0,269 & & 0,089 & \\
\hline Paar mit Kind weiblich & 0,002 & & 0,136 & & 0,132 & & 0,046 & & 0,347 & \\
\hline Paar mit Kleinkind & 0,073 & & $-0,134$ & & $-0,167$ & & $-0,267$ & & $-0,279$ & \\
\hline Paar mit Kleinkind*weiblich & $-0,007$ & & $-0,255$ & & 0,345 & & $-0,011$ & & 0,280 & \\
\hline Alleinerziehend & 0,386 & & 0,902 & & 0,678 & & 0,945 & * & 0,839 & * \\
\hline Erwachsenenhaushalt & 0,032 & & $-0,050$ & & $-0,320$ & & $-0,156$ & & $-0,051$ & \\
\hline Erwachsenenhaushalt ${ }^{*}$ weiblich & 0,327 & & 0,721 & $*$ & 0,628 & * & 0,122 & & 0,368 & \\
\hline Erwachsenes Kind & $-0,046$ & & 0,311 & & 0,452 & & $-0,030$ & & 0,367 & \\
\hline Großeltern & & & 20,079 & & 19,962 & & 0,105 & & $-2,293$ & \\
\hline
\end{tabular}


Tab. 2 Logit-Regression der Pkw-Nutzung als Fahrerin/Fahrer in vollmotorisierten Haushalten. (Eigene Berechnung nach KONTIV/MiD 1976-2008) (Fortsetzung)

\begin{tabular}{|c|c|c|c|c|c|c|c|c|c|c|}
\hline & 1976 & & 1982 & & 1989 & & 2002 & & 2008 & \\
\hline & B & Sign. & $\mathrm{B}$ & Sign. & $\mathrm{B}$ & Sign. & $\mathrm{B}$ & Sign. & $\mathrm{B}$ & Sign. \\
\hline \multicolumn{11}{|c|}{ Gemeindegröße des Wohnortes, Referenz: Gemeinde unter 5 TEW } \\
\hline 5000-20.000 Einwohner & $-0,480$ & & 0,052 & & 0,283 & & 0,100 & & $-0,127$ & \\
\hline $\begin{array}{l}5000-20.000 \\
\text { Einwohner }{ }^{*} \text { weiblich }\end{array}$ & 0,290 & & 0,246 & & $-0,113$ & & $-0,172$ & & $-0,077$ & \\
\hline 20.000-100.000 Einwohner & $-0,431$ & & 0,056 & & $-0,088$ & & 0,149 & & $-0,115$ & \\
\hline $\begin{array}{l}20.000-100.000 \\
\text { Einwohner* weiblich }\end{array}$ & 0,475 & & $-0,060$ & & 0,265 & & $-0,429$ & & 0,030 & \\
\hline 100.000-500.000 Einwohner & $-0,717$ & * & $-0,146$ & & $-0,224$ & & $-0,151$ & & $-0,206$ & \\
\hline $\begin{array}{l}100.000-500.000 \\
\text { Einwohner* weiblich }\end{array}$ & 0,840 & & 0,515 & & $-0,121$ & & $-0,196$ & & $-0,072$ & \\
\hline Ab 500.000 Einwohner & $-0,493$ & & $-0,092$ & & $-0,342$ & & $-0,480$ & $*$ & $-0,446$ & * \\
\hline $\begin{array}{l}\text { Ab } 500.000 \\
\text { Einwohner }{ }^{*} \text { weiblich }\end{array}$ & 0,548 & & 0,429 & & 0,109 & & $-0,399$ & & $-0,174$ & \\
\hline Nagelkerke's $\mathrm{R}^{2}$ & 0,10 & & 0,07 & & 0,08 & & 0,07 & & 0,06 & \\
\hline$N$ (ungewichtet) & 6414 & & 6762 & & 6947 & & 12.020 & & 10.057 & \\
\hline
\end{tabular}

Volljährige am Stichtag mobile Personen, deutsch, alte Bundesländer, FührerscheinbesitzerInnen aus vollmotorisierten Haushalten *Signifikant auf 5\%-Niveau nach Bonferroni-Korrektur, korrigiertes Signifikanzniveau $=0,1 \%$

Tab. 3 Logit-Regression der Pkw-Nutzung als Fahrerin/Fahrer in teilmotorisierten Haushalten. (Eigene Berechnung nach KONTIV/MiD 1976-2008)

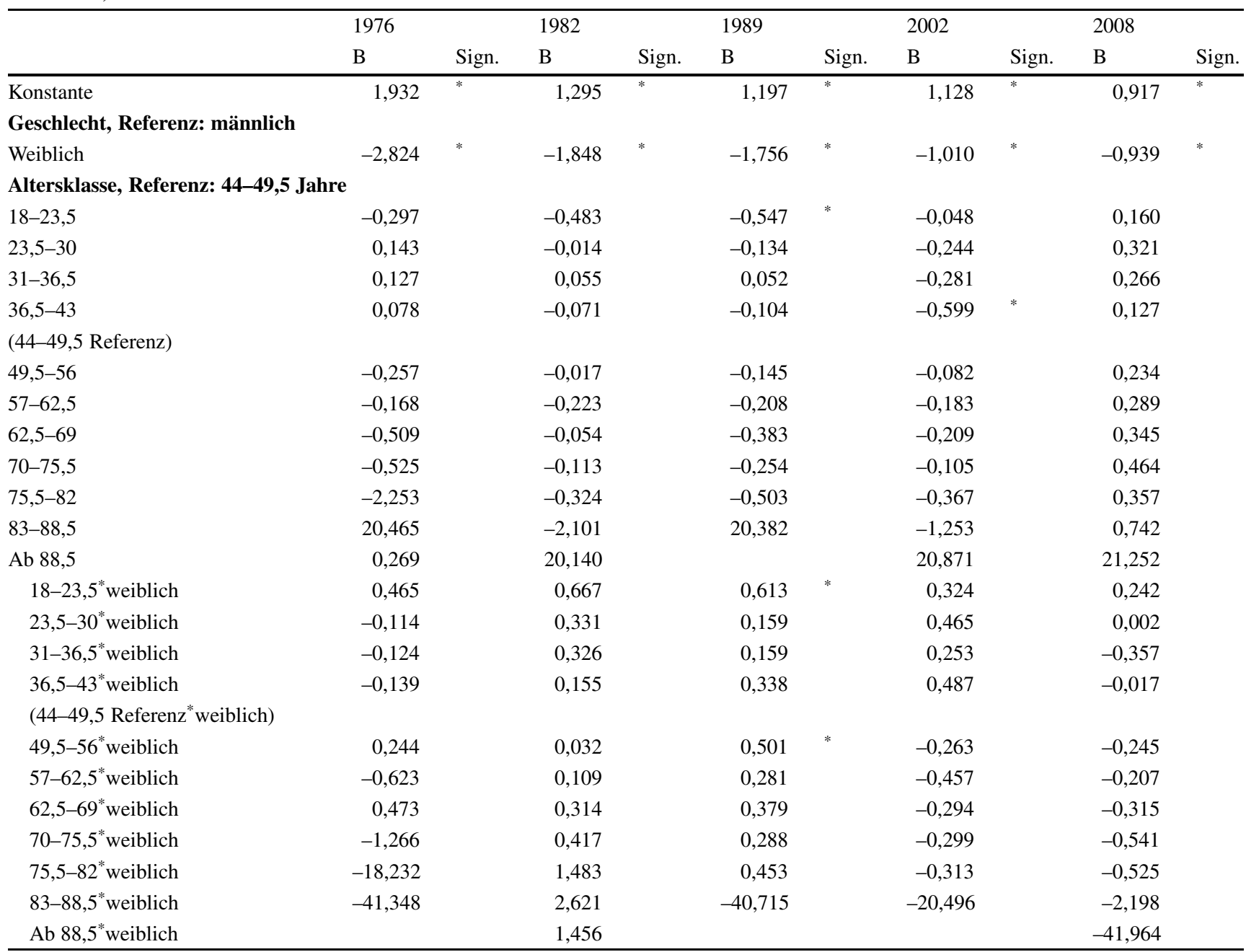


Tab. 3 Logit-Regression der Pkw-Nutzung als Fahrerin/Fahrer in teilmotorisierten Haushalten. (Eigene Berechnung nach KONTIV/MiD 1976-2008) (Fortsetzung)

\begin{tabular}{|c|c|c|c|c|c|c|c|c|c|c|}
\hline & 1976 & & 1982 & & 1989 & & 2002 & & 2008 & \\
\hline & $\mathrm{B}$ & Sign. & B & Sign. & $\mathrm{B}$ & Sign. & B & Sign. & $\mathrm{B}$ & Sign. \\
\hline \multicolumn{11}{|c|}{ Tätigkeit, Referenz: Vollzeiterwerbstätige } \\
\hline Nicht erwerbstätig & $-0,932$ & $*$ & $-0,796$ & * & $-0,585$ & $*$ & $-0,301$ & & $-0,360$ & \\
\hline Nicht erwerbstätig ${ }^{*}$ weiblich & 0,754 & & 0,520 & & 0,205 & & 0,178 & & $-0,041$ & \\
\hline Teilzeit & $-0,851$ & & $-0,277$ & & $-0,507$ & & $-0,374$ & & $-0,266$ & \\
\hline Teilzeit* weiblich & 0,748 & & 0,194 & & 0,299 & & 0,630 & & 0,351 & \\
\hline Ausbildung & $-0,406$ & & $-0,588$ & * & $-1,323$ & * & $-0,900$ & * & $-0,908$ & * \\
\hline Ausbildung*weiblich & 0,303 & & 0,401 & & 0,644 & * & 0,622 & & 0,432 & \\
\hline \multicolumn{11}{|c|}{ Höchster Schulabschluss (für Personen nicht mehr in Ausbildung), Referenz: niedriger als Abitur } \\
\hline Abitur & 0,010 & & $-0,022$ & & 0,160 & & $-0,164$ & & $-0,254$ & \\
\hline Abitur*weiblich & 0,336 & & 0,359 & & 0,285 & & 0,241 & & 0,186 & \\
\hline \multicolumn{11}{|c|}{ Personentyp/Haushaltskontext, Referenz: Paare ohne Kind } \\
\hline Paar mit Kind & $-0,240$ & & 0,010 & & 0,283 & $*$ & 0,037 & & 0,149 & \\
\hline Paar mit Kind ${ }^{*}$ weiblich & 0,773 & * & 0,302 & & 0,486 & * & 0,443 & & 0,662 & * \\
\hline Paar mit Kleinkind & 0,109 & & 0,162 & & 0,111 & & $-0,200$ & & $-0,417$ & \\
\hline Paar mit Kleinkind*weiblich & $-0,197$ & & $-0,371$ & & $-0,312$ & & 0,069 & & 0,315 & \\
\hline Erwachsenenhaushalt & $-0,452$ & & $-0,040$ & & 0,102 & & 0,376 & & 0,219 & \\
\hline Erwachsenenhaushalt* weiblich & 1,093 & * & 0,569 & $*$ & 0,427 & * & 0,202 & & 0,256 & \\
\hline Erwachsenes Kind & $-0,429$ & & 0,213 & & 0,616 & * & 0,330 & & 0,248 & \\
\hline Großeltern & 1,390 & & $-1,335$ & & $-2,414$ & & $-1,006$ & & 0,388 & \\
\hline \multicolumn{11}{|c|}{ Gemeindegröße des Wohnortes, Referenz: Gemeinde unter 5 TEW } \\
\hline 5000-20.000 Einwohner & $-0,274$ & & $-0,016$ & & $-0,004$ & & $-0,063$ & & $-0,173$ & \\
\hline $\begin{array}{l}\text { 5000-20.000 } \\
\text { Einwohner }{ }^{*} \text { weiblich }\end{array}$ & 0,384 & & $-0,082$ & & $-0,198$ & & 0,035 & & 0,049 & \\
\hline 20.000-100.000 Einwohner & $-0,343$ & & 0,105 & & $-0,243$ & & 0,017 & & $-0,251$ & \\
\hline $\begin{array}{l}20.000-100.000 \\
\quad \text { Einwohner* weiblich }\end{array}$ & 0,407 & & $-0,095$ & & $-0,092$ & & $-0,259$ & & $-0,090$ & \\
\hline 100.000-500.000 Einwohner & $-0,098$ & & 0,085 & & $-0,225$ & & 0,083 & & $-0,431$ & * \\
\hline $\begin{array}{l}100.000-500.000 \\
\text { Einwohner* weiblich }\end{array}$ & 0,177 & & $-0,405$ & & $-0,298$ & & $-0,673$ & * & $-0,126$ & \\
\hline Ab 500.000 Einwohner & $-0,426$ & & $-0,375$ & & $-0,150$ & & $-0,331$ & & $-0,648$ & $*$ \\
\hline Ab 500.000 Einwohner* weiblich & 0,260 & & 0,169 & & $-0,339$ & & $-0,501$ & & $-0,224$ & \\
\hline Nagelkerke's $\mathrm{R}^{2}$ & 0,28 & & 0,18 & & 0,21 & & 0,11 & & 0,11 & \\
\hline$N$ (ungewichtet) & 8348 & & 8703 & & 10.921 & & 7595 & & 9674 & \\
\hline
\end{tabular}

Volljährige am Stichtag mobile Personen, deutsch, alte Bundesländer, FührerscheinbesitzerInnen aus teilmotorisierten Haushalten

*Signifikant auf 5\%-Niveau nach Bonferroni-Korrektur, korrigiertes Signifikanzniveau $=0,1 \%$

Ressource Pkw in Familien intensiver genutzt als in Haushalten ohne Kinder. Die Umverteilung des Pkw als knappe Ressource zwischen Männern und Frauen im Zeitverlauf zeigt sich ähnlich stark in allen Paarhaushalten (keine systematische Veränderung von Koeffizienten in Tab. 3). Die Konvergenz ist unter den hier modellierten Rahmenbedingungen in Familien bis 2008 am weitesten vorangeschritten, es besteht kein nennenswerter Unterschied mehr zwischen Müttern und Vätern. Das gilt aber nur bei Kontrolle relevanter Rahmenbedingungen (z. B. Vollzeiterwerbstätigkeit). In der Realität sind diese Rahmenbedingungen gerade in Familien ihrerseits stark ungleich zwischen Männern und Frauen verteilt, sodass in deskriptiver Betrachtung auch in Familien bis 2008 noch deutliche Geschlechterunterschie- de der Pkw-Nutzung zu beobachten sind (vgl. auch Manz/ Bauer/Herget et al. 2015; Konrad 2016: 184 ff.).

Der Wohnort (Gemeindegrößenklasse) hat zwar kaum einen signifikanten Effekt auf die Pkw-Nutzung (vgl. Tab. 2 und 3), doch zeigen sich raumspezifische Trends, die in den Beispielrechnungen herausstechen (vgl. Abb. 7 und 8). Die zunehmende Pkw-Nutzung der Frauen aus teilmotorisierten Haushalten ist insbesondere in kleinen und mittleren Städten zu beobachten, deutlich stärker als in Großstädten (vgl. Abb. 8). Die komplexer werdenden Alltagsmuster speziell der Frauen sind offenbar gerade in Räumen mit geringem ÖPNV-Angebot und langen Alltagsdistanzen kaum ohne Pkw zu bewältigen. 


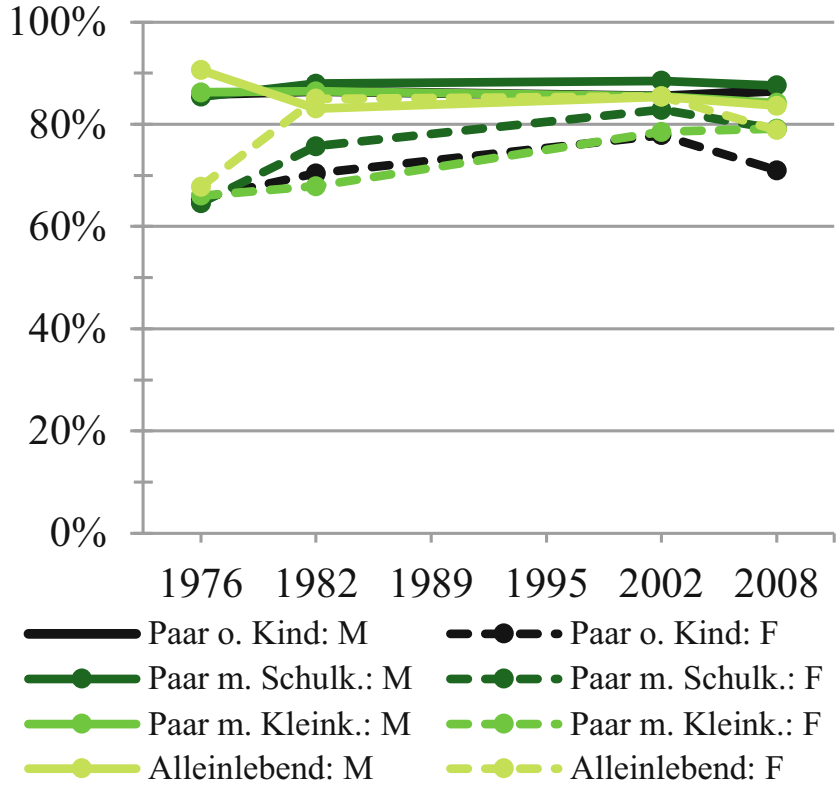

Abb. 5 Pkw-Nutzung als Fahrerin/Fahrer in vollmotorisierten Haushalten differenziert nach Haushaltskontext (Gruppen: vollzeiterwerbstätig, 44-49,5 Jahre, ohne Abitur, Paar ohne Kind/Paar mit Schulkind/ Paar mit Kleinkind/alleinlebend, Wohnort 5000 bis $<20.000$ Einwohner, Vollmotorisiert: mindestens so viele Pkw wie fahrfähige Personen im Haushalt)

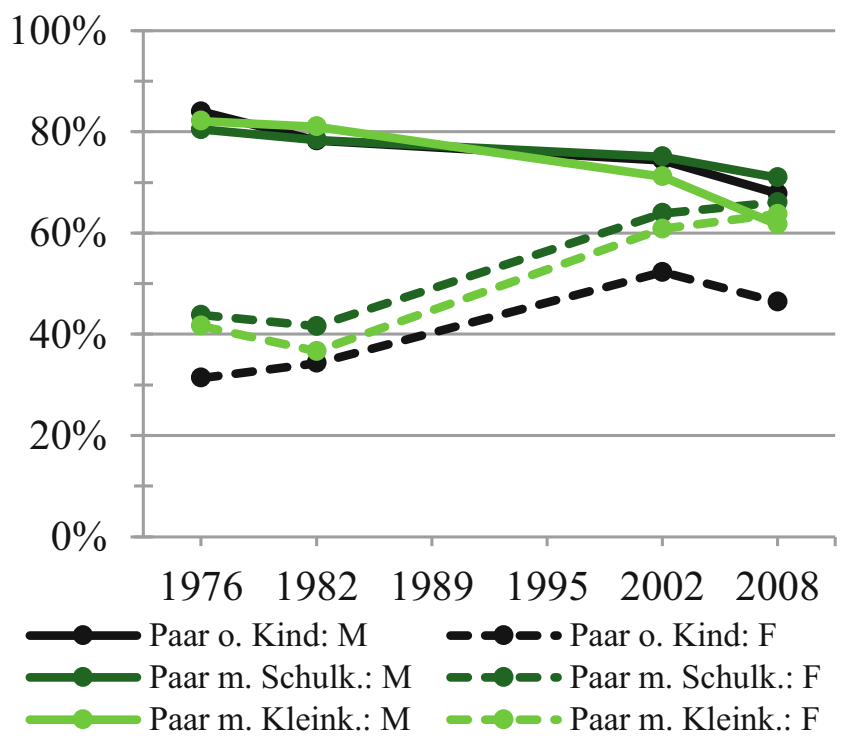

Abb. 6 Pkw-Nutzung als Fahrerin/Fahrer in teilmotorisierten Haushalten differenziert nach Haushaltskontext (Gruppen: vollzeiterwerbstätig, 44-49,5 Jahre, ohne Abitur, Paar ohne Kind/Paar mit Schulkind/ Paar mit Kleinkind/alleinlebend, Wohnort 5000 bis $<20.000$ Einwohner, Teilmotorisiert: weniger Pkw als fahrfähige Personen im Haushalt)

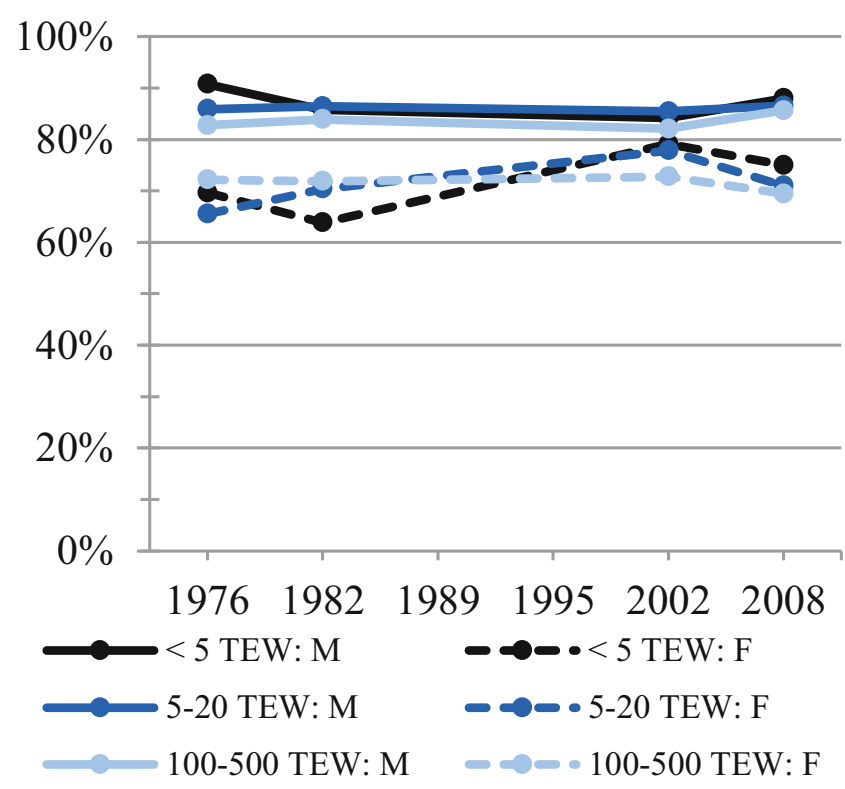

Abb. 7 Pkw-Nutzung als Fahrerin/Fahrer in vollmotorisierten Haushalten differenziert nach Gemeindegröße (Gruppen: Vollzeiterwerbstätig, 44-49,5 Jahre, ohne Abitur, Paar ohne Kind, Wohnort <5000 Einwohner/5000 bis $<20.000$ Einwohner/100.000 bis $<500.000$ Einwohner, Vollmotorisiert: mindestens so viele Pkw wie fahrfähige Personen im Haushalt)

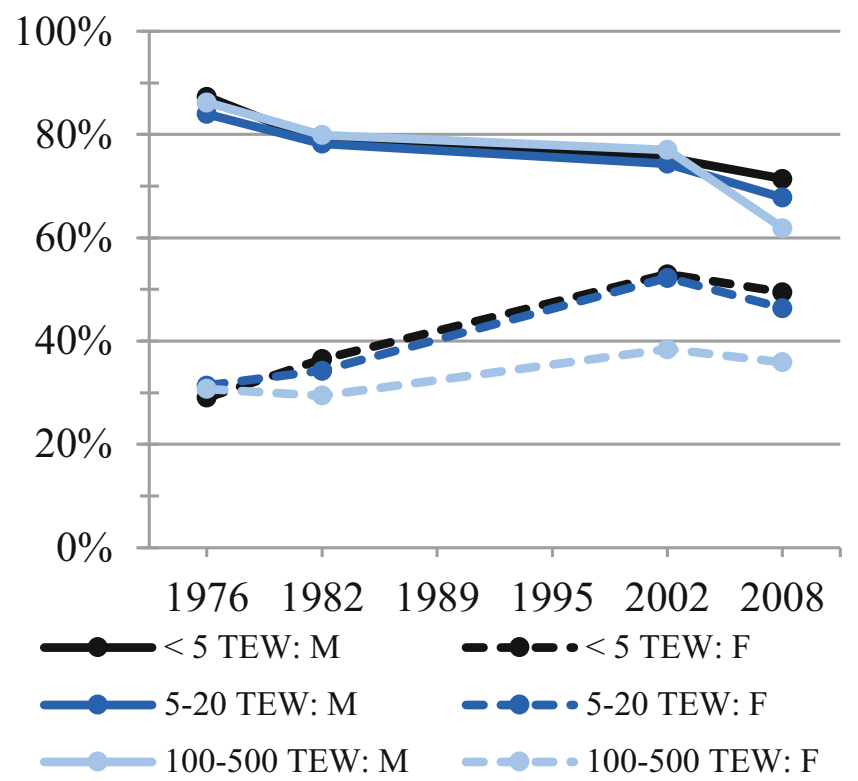

Abb. 8 Pkw-Nutzung als Fahrerin/Fahrer in teilmotorisierten Haushalten differenziert nach Gemeindegröße (Gruppen: Vollzeiterwerbstätig, 44-49,5 Jahre, ohne Abitur, Paar ohne Kind, Wohnort <5000 Einwohner/5000 bis $<20.000$ Einwohner/100.000 bis $<500.000$ Einwohner, Teilmotorisiert: weniger Pkw als fahrfähige Personen im Haushalt) 


\section{Zusammenfassung und Ausblick}

Dies ist unseres Wissens die erste Studie zu diesem Thema „Pkw-Nutzung im Wandel des Geschlechtsverhältnisses" weltweit, die einen langen Zeitraum von über drei Jahrzehnten abbildet, Geschlechterverhalten systematisch in weitere soziale Strukturen einbettet und damit neben dem Nachzeichnen langfristiger Trends eine Einschätzung zu den theoretischen Annahmen über die Beziehung zwischen Geschlecht und Alltagsmobilität erlaubt. Hinsichtlich der zuvor formulierten Fragestellungen sind folgende zentrale Ergebnisse festzuhalten:

- Die Analysen zeigen, dass die Pkw-Nutzung von Männern und Frauen zwischen 1976 und 2008 deutlich konvergiert. In erster Linie resultiert dies aus einer Annäherung des Verhaltens der Frauen an das der Männer, also einer Art Aufholprozess. Dies gilt aber nicht ausschließlich. In teilmotorisierten Haushalten, in denen der Pkw eine knappe Ressource darstellt, nimmt die Pkw-Nutzung der Männer ab. Hier findet also eine Umverteilung statt.

- Die Umverteilung des Pkw in teilmotorisierten Haushalten lässt sich nicht allein durch die Konvergenz von Rahmenbedingungen der Mobilität, etwa die zunehmende Frauenerwerbstätigkeit (Rollentheorie), Führerscheinausstattung und Pkw-Verfügbarkeit (Restriktionstheorie), erklären. Die Berechnungen aus den Regressionsmodellen beziehen sich auf vollzeiterwerbstätige Männer und Frauen mit Fahrerlaubnis und gleicher PkwAusstattung. Hier scheint ein Wandel von Normen relevant, der sich auch in der Alltagsmobilität niederschlägt.

- Selbst in vollmotorisierten Haushalten fahren im Zeitverlauf die Frauen immer häufiger Auto (obwohl sie das in diesen Haushalten auch schon 1976 hätten tun können). Auch dies spricht für einen Normenwandel (der anhand der vorliegenden Daten empirisch nicht direkt belegt werden kann) oder eine generell zunehmende Pkw-Abhängigkeit selbst derjenigen, die bereits in den 1970erJahren über ein Auto verfügten.

- Anhand der Analysen wird deutlich, dass soziale Rollen und konkret die Arbeitsteilung die geschlechtsspezifische Alltagsmobilität, hier die Pkw-Nutzung, beeinflussen. Die Ergebnisse verdeutlichen wie folgt die Relevanz der Rollentheorie, die über die Erwerbstätigkeit und den Haushaltskontext überprüft wurde, für geschlechtsspezifische Unterschiede in der Pkw-Nutzung: Erstens geht ein höherer Erwerbsumfang mit einer häufigeren Pkw-Nutzung einher. Bei Frauen sind im Schnitt die Erwerbsbeteiligung und der Erwerbsumfang niedriger als bei Männern, was sich in der einleitenden deskriptiven Analyse in einer auffallend niedrigeren Pkw-Nutzung niederschlägt. Zum anderen wirkt sich die geschlechts- spezifische Komplexität von Rollen und damit von Aktivitätsmustern auf die Pkw-Nutzung aus: je komplexer der Alltag, desto wahrscheinlicher die Autonutzung. So haben erwerbstätige Mütter laut zahlreicher Studien einen äußerst komplexen Alltag und nutzen, speziell in teilmotorisierten Haushalten, häufiger als andere erwerbstätige Frauen den Pkw.

- Die deutlich größeren Unterschiede zwischen Männern und Frauen in teilmotorisierten Haushalten weisen auf die Relevanz von rational-ökonomischen Haushaltsentscheidungen bzw. haushaltsinternen Aushandlungen hin. Bis 2008 bestehen Disparitäten bei der Verfügung über die knappe Ressource Pkw. Schwächere Verhandlungspositionen der Frauen können beispielsweise mit ihrem niedrigeren Durchschnittseinkommen zusammenhängen, das hier nicht empirisch kontrolliert werden konnte. An der starken Konvergenz wird aber der Bedeutungsverlust solcher rational-ökonomischer Aushandlungen deutlich.

- Über rollentheoretische und rational-ökonomische Erklärungsansätze (geschlechtsspezifische Restriktionen wurden über die Unterteilung in teil- und vollmotorisierter Haushalte ausgeklammert) gehen die geschlechtsspezifischen Unterschiede in der Pkw-Nutzung aber noch hinaus: Denn selbst in vollmotorisierten Mehrpersonenhaushalten fahren Frauen bei gleichem Erwerbsumfang signifikant seltener Auto als die Männer. Das galt 2008 genauso wie 1976. In Teilen könnte das aus gemeinsam zurückgelegten Wegen resultieren, bei denen der Mann fährt und die Frau mitfährt. Dabei stellt sich die Frage nach dem Grund für diese Verteilung. Offenbar spielen Aspekte abseits von sozialen Rollen, Restriktionen und rational-ökonomisch geprägten Aushandlungen eine Rolle für die seltenere Autonutzung der Frauen. In der Literatur werden dazu passend etwa die stärkere Technikbegeisterung von Männern sowie deren Macht in Partnerschaften diskutiert, Steuerungspositionen (hier im wörtlichen Sinne) einzunehmen (Polk 1998; Law 1999). Jedoch können auch, wie von Matthies, Kuhn und Klöckner (2002: 165 f.) beschrieben, geschlechtsspezifische Präferenzen in Form stärkerer ökologischer Normen, einer geringeren Pkw-Affinität oder schwächerer Gewohnheitsprägungen der Verkehrsmittelnutzung bei Frauen eine Rolle spielen, die in der feministisch geprägten Forschung zu Geschlechterunterschieden der Mobilität weniger häufig vertreten und entsprechend weniger untersucht werden.

Die Ergebnisse bestätigen über einen langen Betrachtungszeitraum hinweg weitgehend, was zahlreiche ältere Querschnittsstudien herausfinden: Frauen fahren seltener als Männer Auto. Auch die Ergebnisse der wenigen bisherigen Trendanalysen zur Pkw-Nutzung im Geschlechterverhältnis können bestätigt werden: Trotz eines „Aufholens“ der 
Frauen bleibt ihre Pkw-Nutzung (bis 2008) niedriger als die der Männer. Speziell unter jungen Erwachsenen hat sich dagegen in den letzten Jahren eine weitgehende Angleichung vollzogen (Kuhnimhof/Armoogum/Buehler et al. 2012), und es bleibt abzuwarten, inwieweit sich diese als Kohorteneffekt zukünftig in höhere Altersgruppen fortschreiben wird.

Über den bisherigen Forschungsstand gehen unsere Ergebnisse aber deutlich hinaus. So ermöglichen sie anhand empirischer Befunde und mittels differenzierter Analysen Rückschlüsse auf die Aussagekraft theoretischer Erklärungsansätze für geschlechtsspezifische Mobilität, hier die Pkw-Nutzung. Außerdem zeigen sie Trends in der PkwNutzung im Geschlechterverhältnis über einen langen Zeitraum und nach soziodemographischen und räumlichen Merkmalen differenziert.

Für die weitere Forschung lassen sich eine Reihe von Schlussfolgerungen ziehen. Erstens sind unsere empirischen Messungen der theoretischen Hypothesen datenbedingt grob. Soziale Rollen, Restriktionen und ökonomische Rationalitäten unterliegen sehr viel komplexeren Grundlagen und Mechanismen als sich mit wenigen sozioökonomischen und demographischen Rahmenbedingungen abbilden lassen. Über Geschlechternormen, Einstellungen und Mobilitätspräferenzen haben wir keine direkten Informationen. Auch individuelle Einkommensinformationen fehlen. Wichtig wäre in der zukünftigen Forschung vor allem die Kontrolle des persönlichen Beitrags zum jeweiligen Haushaltseinkommen sowie von Normen und Einstellungen, um das Verhältnis ökonomisch bestimmter Machtrelationen und persönlicher Verhaltenspräferenzen besser ausloten zu können. Aber auch die Beiträge zweier Partner zur Familien- und Haushaltsarbeit (etwa Begleitwege, Einkauf, häusliche Aktivität) wären von Bedeutung, um soziale Rollen besser zu verstehen.

Zweitens ließen sich auch die räumlichen Rahmenbedingungen in der hier untersuchten langen Zeitreihe nur sehr grob klassifizieren. In drei Jahrzehnten haben sich die Lebensstile und sozialen Strukturen und damit die Geschlechterverhältnisse in Stadt und Land unterschiedlich entwickelt.

Drittens würden solche differenzierten Analysen eine vertiefte Auseinandersetzung mit der sozialen Dimension des beobachteten Verhaltens erlauben. Unsere Analysen zeigen eine deutliche Konvergenz der Mobilität von Frauen und Männern im Lauf der letzten Jahrzehnte. Dies spricht für eine zunehmende Gleichheit von Möglichkeiten und Verhalten. Dem stehen die negativen Umweltauswirkungen des Autos sowie die Exklusionsmechanismen gegenüber, die das Auto für diejenigen produziert, die auch in einer hochmotorisierten Gesellschaft nicht darauf zurückgreifen können. Zudem lässt sich anhand von verhaltensorientierten Studien nicht klären, inwieweit beobachtete Unterschiede auf unterschiedlichen Chancen oder bloßen Vorlieben beruhen. Weitere Forschungsbeiträge zur Klärung dieser Fragen könnten auch die planerisch-politische Debatte um Geschlechterverhältnisse und das Auto anregen.

\section{Literatur}

van Acker, V.; Witlox, F. (2011): Commuting trips within tours: How is commuting related to land use? In: Transportation 38, 3, 465-486.

Anger, S.; Kottwitz, A. (2009): Mehr Hausarbeit, weniger Verdienst. In: DIW Wochenbericht 76, 6, 102-109.

Bamberg, S. (1996): Zeit und Geld: Empirische Verhaltenserklärung mittels Restriktionen am Beispiel der Verkehrsmittelwahl. In: ZUMA-Nachrichten 20, 38, 7-32.

Beck, U. (1986): Risikogesellschaft. Auf dem Weg in eine andere Moderne. Frankfurt am Main: Suhrkamp.

Best, H.; Lanzendorf, M. (2005): Division of labour and gender differences in metropolitan car use. An empirical study in Cologne, Germany. In: Journal of Transport Geography 13, 2, 109-121.

Bland, J.M.; Altman, D.G. (1995): Multiple significance tests: the Bonferroni method. In: British Medical Journal 310, 6973, 170.

BMFSFJ - Bundesministerium für Familie, Senioren, Frauen und Jugend (2011): Neue Wege - Gleiche Chancen. Gleichstellung von Frauen und Männern im Lebensverlauf. Erster Gleichstellungsbericht. Berlin.

Brennecke, J. (1994): Alltagsmobilität Wiesbadener Frauen. In: Apel, D.; Holzapfel, H.; Kiepe, F.; Lehmbrock, M.; Müller, P. (Hrsg.): Handbuch der kommunalen Verkehrsplanung, 6. Ergänzungslieferung. Bonn, 1-22.

Buhr, R. (1999): Neue Nutzungskontexte. Zur Bedeutung des Autos im Alltag von Frauen. In: Buhr, R.; Canzler, W.; Knie, A.; Rammler, S. (Hrsg.): Bewegende Moderne. Fahrzeugverkehr als soziale Praxis. Berlin, 105-131.

Choo, S.; Mokhtarian, P.L. (2004): What type of vehicle do people drive? The role of attitude and lifestyle in influencing vehicle type choice. In: Transportation Research Part A 38, 3, 201-222.

Dobbs, L. (2005): Wedded to the car: women, employment and the importance of private transport. In: Transport Policy 12, 3, 266-278.

Dressel, C. (2005): Erwerbstätigkeit - Arbeitsmarktintegration von Frauen und Männern. In: Cornelißen, W. (Hrsg.): Gender-Datenreport. 1. Datenreport zur Gleichstellung von Frauen und Männern in der Bundesrepublik Deutschland. München, 99-158.

Flade, A. (1999): Zu den Ursachen des unterschiedlichen Mobilitätsverhaltens von Männern und Frauen. In: Flade, A.; Limbourg, M. (Hrsg.): Frauen und Männer in der mobilen Gesellschaft. Opladen, $137-151$.

Götz, K. (2011): Nachhaltige Mobilität. In: Groß, M. (Hrsg.): Handbuch Umweltsoziologie. Wiesbaden, 325-347.

Hagman, O. (2003): Mobilizing meanings of mobility: car users' constructions of the goods and bads of car use. In: Transportation Research Part D 8, 1, 1-9.

Hamilton, K.; Jenkins, L. (2000): A Gender Audit for Public Transport: A New Policy Tool in the Tackling of Social Exclusion. In: Urban Studies 37, 10, 1793-1800.

Hensher, D.A.; Reyes, A.J. (2000): Trip chaining as a barrier to the propensity to use public transport. In: Transportation 27, 4, 341-361.

Hjorthol, R. (2008): Daily Mobility of Men and Women - A Barometer of Gender Equality? In: Uteng, T.P.; Cresswell, T. (Hrsg.): Gendered Mobilities. Aldershot, 193-209.

Hurrelmann, K. (2012): Kindheit, Jugend und Gesellschaft. Identität in Zeiten des schnellen sozialen Umbruchs - soziologische Perspektiven. In: Petzold, H.G. (Hrsg.): Identität. Wiesbaden, 57-75.

Klammer, U. (2012): Hochgebildet - ausgebremst - (re)aktiviert alimentiert: Frauenerwerbsverläufe in Deutschland. Ein Diskussionsbeitrag zum Thema „Fachkräftemangel. In: Bispinck, R.; 
Bosch, G.; Hofemann, K.; Naegele, G. (Hrsg.): Sozialpolitik und Sozialstaat. Wiesbaden, 163-188.

Konrad, K. (2016): Mobiler Alltag im Wandel des Geschlechterverhältnisses. Wiesbaden. = Studien zur Mobilitäts- und Verkehrsforschung 29.

Kuhnimhof, T.; Armoogum, J.; Buehler, R.; Dargay, J.; Denstadli, J.M.; Yamamoto, T. (2012): Men Shape a Downward Trend in Car Use among Young Adults - Evidence from Six Industrialized Countries. In: Transport Reviews 32, 6, 761-779.

Law, R. (1999): Beyond 'women and transport': towards new geographies of gender and daily mobility. In: Progress in Human Geography $23,4,567-588$.

Liebig, S.; Sauer, C.; Schupp, J. (2011): Die wahrgenommene Gerechtigkeit des eigenen Erwerbseinkommens: Geschlechtstypische Muster und die Bedeutung des Haushaltskontextes. In: Kölner Zeitschrift für Soziologie und Sozialpsychologie 63, 1, 33-59.

Maiwald, K.-O. (2012): Modern und doch traditional? Paradoxien heutiger Paarbeziehungen. In: Soeffner, H.-G. (Hrsg.): Transnationale Vergesellschaftungen. Wiesbaden, 911-918.

Manz, W.; Bauer, U.; Herget, M.; Scheiner, J. (2015): Familienmobilität im Alltag. Endbericht des Projekts ,Determinanten und Handlungsansätze der Familienmobilität“. Berlin.

Matthies, E.; Kuhn, S.; Klöckner, C.A. (2002): Travel Mode Choice of Women: The Result of Limitation, Ecological Norm, or Weak Habit? In: Environment and Behavior 34, 2, 163-177.

Meuser, M. (2008): Geschlechter und Geschlechterverhältnisse. In: Willems, H. (Hrsg.): Lehr(er)buch Soziologie. Für die pädagogischen und soziologischen Studiengänge. Wiesbaden, 631-653.

Meuser, M. (2009): Männer und Familie - Perspektiven aus der Männlichkeitsforschung. In: Kapella, O.; Rille-Pfeiffer, C.; Rupp, M.; Schneider, N.F. (Hrsg.): Die Vielfalt der Familie. Tagungsband zum 3. Europäischen Fachkongress Familienforschung. Opladen/ Farmington Hills, 145-155.

Meyer, H. (1999): Frauenmobilität - Männermobilität? Ergebnisse einer Untersuchung in der schweizerischen Stadt Zürich. In: Internationales Verkehrswesen 51, 1/2, 25-30.

Nobis, C. (2008): Geschlechtsspezifische Unterschiede im Mobilitätsverhalten unter besonderer Berücksichtigung der Aktivi- tät Einkaufen. In: Achen, M.; Böhmer, J.; Gather, M.; Pez, P. (Hrsg.): Handel und Verkehr, Mobilität und Konsum. Mannheim, $105-130$.

Peuckert, R. (2012): Familienformen im sozialen Wandel. Wiesbaden. Polk, M. (1998): Gendered Mobility: A Study of Women's and Men's Relations to Automobility in Sweden. Göteborg.

Polk, M. (2004): The influence of gender on daily car use and on willingness to reduce car use in Sweden. In: Journal of Transport Geography 12, 3, 185-195.

Rosenbloom, S. (1978): The Need for Study of Women's Travel Issues. In: Transportation 7, 4, 347-350.

Rutherford, B.; Wekerle, G.R. (1988): Captive Rider, Captive Labor: Spatial Constraints and Women's Employment. In: Urban Geography 9, 2, 116-137.

Schäfer, A.; Vogel, C. (2005): Teilzeitbeschäftigung als Arbeitsmarktchance. In: DIW Wochenbericht 72, 7, 131-138.

Scheiner, J. (2009): Sozialer Wandel, Raum und Mobilität. Empirische Untersuchungen zur Subjektivierung der Verkehrsnachfrage. Wiesbaden.

Scheiner, J.; Holz-Rau, C. (2012): Gendered travel mode choice: a focus on car deficient households. In: Journal of Transport Geography $24,250-261$.

Schendera, C.F.G. (2008): Regressionsanalyse mit SPSS. München.

Schubarth, W. (2010): Die „Rückkehr der Werte“. Die neue Wertedebatte und die Chancen der Wertebildung. In: Schubarth, W.; Speck, K.; von Berg, H.L. (Hrsg.): Wertebildung in Jugendarbeit, Schule und Kommune. Bilanz und Perspektiven. Wiesbaden, 21-41.

Shell Deutschland Oil (2014): Shell PKW-Szenarien bis 2040. Fakten, Trends und Perspektiven für Auto-Mobilität. Hamburg.

Valley, H. (2003): Mobilität und Verkehr. In: Gebhardt, H.; Warneken, B.J. (Hrsg.): Stadt - Land - Frau. Interdisziplinäre Genderforschung in Kulturwissenschaft und Geographie. Heidelberg, 64-95. = Heidelberger geographische Arbeiten 117.

Vance, C.; Iovanna, R. (2007): Gender and the Automobile. Analysis of Non-work Service Trips. Essen. = Ruhr Economic Papers 11. 